\title{
TAHSP :
}

The Internet Joưnal of Allied Health Sciences and Practice

A Peer Reviewed Publication of the College of Health Care Sciences at Nova Southeastern University

Dedicated to allied health professional practice and education

http://ijahsp.nova.edu Vol. 12 No. 3 ISSN 1540-580X

\section{Health Promotion Strategies Derived from a Metropolitan Police Weight Loss Competition with Weight Loss Comparisons by Gender and BMI Category}

\author{
Robert Boyce, PhD, FACSM ${ }^{1}$ \\ Tyler Willett, BS2 \\ Allen Mullins, BS2 \\ Glenn Jones, MS, MPA4 \\ Randy Cottrell, DEd, MCHES 5
}

1. Associate Professor, School of Health and Applied Human Sciences, University of North Carolina Wilmington, Wilmington, NC

2. Student Research Assistant, School of Health and Applied Human Sciences, University of North Carolina Wilmington, Wilmington, NC

3. Physical Fitness Coordinator, Charlotte-Mecklenburg Police Department, Work Physiology Associates, Inc., Charlotte, NC

4. Professor, School of Health and Applied Human Sciences, University of North Carolina Wilmington, Wilmington, NC

United States

CITATION: Boyce R, Willett T, Mullins A, Jones, G, Cottrell R. Health Promotion Strategies Derived from a Metropolitan Police Weight Loss Competition with Weight Loss Comparisons by Gender and BMI Category. The Internet Journal of Allied Health Sciences and Practice. July 2014. Volume 12 Number 3.

\section{ABSTRACT}

Background: Weight management is critical in police work. It can have both legal and life-saving implications as it is linked to the health and physical readiness of the officers. A weight loss competition is one way to address weight management in police work. Among the factors to consider is gender impact, as females forge forward within a male environment. Also, assessing weight loss among obesity categories is essential for designing effective programing. Purpose: To compare mass changes among body mass index (BMI) categories and between genders at biweekly weigh-ins during a 12-week competition with recommendations for professionals. Methods: Before the competition began, the participants were instructed that those teams recording greatest percentage loss won. Body mass (body weight) comparisons were made between genders and among BMI categories. Results: Mean body mass lost for the 225 officers was $5.26 \mathrm{~kg}$. Genders had similar mass losses. Normal and overweight BMI classes had significantly $(p \leq 0.05)$ less body mass changes than obese classes. In weeks 2 to 4 , the extreme obese category experienced greatest losses. No differences were found in amount of loss among any of the groups from weeks 6 to 12. Conclusion: This multiple-site, large-scale and low-cost intervention specific to police was successful. The program had equivalent gender impact in terms of mass lost. In the second half of the program, the higher BMI groups did not lose significantly more than the lower BMI groups indicating physiological and/or motivational effects to be addressed in future programs.

\section{INTRODUCTION}

Obesity is reported to be at epidemic proportions in western societies. In the United States, the percentage of those classified as overweight and obese is at a record high, $68.8 \%$, with over $35.7 \%$ being classified as obese. ${ }^{1}$ Obesity is reported to affect all age groups below 70 years. ${ }^{2}$ This raises concerns based on the association of obesity with increases in morbidity and mortality. 
Prevalent disease conditions connected to obesity include cardiovascular disease, type 2 diabetes, gallbladder disease, stroke, osteoarthritis, and some cancers. ${ }^{3}$ Clearly, obesity can have dramatic negative effects on the individual as well as industry and health care systems with corresponding costs and economic issues. ${ }^{4}$

\section{Background}

Studies of work environments have revealed associations between greater body mass index (BMI) and long working hours, shift work, and job stress. ${ }^{5}$ There is also evidence of increased obesity in high demand and low-control work environments. ${ }^{5}$ Police officers are subject to these work conditions. Furthermore, police work differs from other professionals as their job requires periods of inactivity followed by bursts of high activity which maybe in emergency or life threatening situations. This adds to the stress, high demand, and low-control work environment.

Studies have shown that police officers' body mass increased significantly after the initial academy training. ${ }^{6} \mathrm{~A}$ recent longitudinal report on police documented an increase of approximately $0.91 \mathrm{~kg}$ per year for male officers and a $0.68 \mathrm{~kg}$ per year for females in the first decade of employment. ${ }^{7}$ Police work involves high stress that is linked to a disproportionate number of health problems, such as heart disease, gastrointestinal disorders, alcoholism, emotional disorders, excess weight, and weight gain. ${ }^{8-10}$ The resulting obesity may potentially affect both work opportunity and job performance. ${ }^{5}$

Employers are keenly interested in worksite-based health promotional programming to effect weight loss while offering opportunities for decreasing adult obesity and health care costs. ${ }^{11,} 12$ Qualitative and quantitative studies have yielded positive results on the effectiveness of worksite initiatives for controlling workers' weight. ${ }^{13}$ Direct and indirect costs to employers are substantial and correspond with rising health insurance premiums. ${ }^{14}$ Additional programming can lead to cost and time savings such as reduced absenteeism, sick leave, disability injuries, and healthcare claims. ${ }^{15-17}$

Over the past 30 to 40 years, research has led to improvements in short-term weight loss program efficacy. ${ }^{12}$ Voluntary weight loss is a common practice in the United States regardless of age, gender, or ethnicity. ${ }^{18}$ Therefore, it makes sense to offer worksite weight loss programming to affect large groups of adults. ${ }^{14}$ For worksites offering weight loss programming, it appears the self-regulated approach (one that includes regular weigh-ins, self-reinforcement, and self-corrective action) has proven to be successful. ${ }^{19}$

Numerous worksite studies address weight loss programs. ${ }^{14,} 20$ However, our review revealed a paucity of recent research regarding in-service police weight loss competitions. Little research was found that compared weight loss patterns between BMI categories and none concerning police. Reports on weight loss among in-service police dealt primarily with exercise and nutrition-based interventions. ${ }^{6,21}$ Little emphasis is placed on patterns of weight loss and how interventions can be modified according to these patterns to enhance efficacy.

In light of the Healthy People 2020 obesity prevention goals, there is a need to explore the viability of weight loss competitions, especially in police departments. ${ }^{22}$ The Charlotte-Mecklenburg Police Department, a law enforcement agency in the Southeastern United States, conducted its first weight loss competition. The purpose of this report is to compare the weight loss of sworn officers among BMI categories and between genders at each of the biweekly weigh-ins during this 12-week weight loss competition, as well as suggest health promotional strategies based on the results and authors' observations of this program. More specifically in this study, the tested null hypothesis was that in a 12-week police weight loss competition, there will be no differences between genders and among BMI categories regarding the amount of mass lost. Also, there will be no changes in the starting to final mean body mass over the 12-week period. Furthermore, there will be no significant differences in weight loss over the 12-week period between genders or among BMI categories.

\section{METHOD}

\section{Participants}

The participants for this study consisted of 225 sworn police officers from the Charlotte-Mecklenburg Police Department that volunteered to participate in a weight loss competition. Approximately, 1,717 police officers were in the Charlotte Mecklenburg Police Department. The University of North Carolina at Wilmington Institutional Review Board, which follows the Office of Human Research Protection Requirements, was contacted. Since this research involved existing and individually unidentifiable data, it was exempt from applicable human subject regulation.

\section{Protocol}

A team weight loss competition was offered to all employees of the Charlotte-Mecklenburg Police Department. The program ran from January 3, 2011 to March 28, 2011. Participants formed their own teams of three to four employees and these teams 
entered the competition. Teams competed against each other as well as amongst the various districts, locations, or departments including the 13 Patrol Districts, the different floors of the Police Headquarters building, Communications, the Academy, and Animal Control. After the initial weigh-in, participants were required to weigh-in every other week throughout the course of the program for a total of seven weigh-ins. The weigh-ins were conducted in each district, location, or department with a Detecto beam-balance scale (detecto.com), and the participants weighed wearing a T-shirt, shorts, and without shoes. The same model and series of Detecto scales were used at each weigh-in location, and individuals were weighed on the same scale each time. Scales were calibrated to zero before each weigh-in. Coordinators were trained on the proper use and management of the scales. Participants who completed all the weigh-ins were eligible for rewards. All participants were required to sign an informed consent waiver.

The police department's fitness coordinator, a master's level exercise physiologist, directed the program. The fitness coordinator reported to an administrator in the police department who served as a liaison to upper level management. Coordinators were designated within each district and their role was to administer and record all weigh-ins, and report these results to the fitness coordinator. The fitness coordinator also wrote and distributed an email newsletter that was sent to participants before each weigh-ins. This newsletter included information about the competition, nutrition, healthy recipes, and exercise. Each district coordinator received training via written instructions on weigh-ins and administrative protocols.

Six weeks before the competition, emails were sent out as a promotional teaser to the employees announcing the competition with additional information being added each week. Two weeks prior to the start of the competition, the process on how to register was provided. The teams registered by email with the district coordinators who gave this information to the fitness coordinator. A complete description of the rules and prizes for the winners was also given to each participant along with how one could obtain personal counseling. Personal counseling was provided by physical fitness instructors at the police academy. Counseling involved individual dietary and fitness information and recommendations. All counselors were state-certified physical fitness instructors through the North Carolina Police Academy.

The competition distributed awards and prizes for the following categories:

- Participation Award (defined as attending all weigh-ins) - Dri-FIT shirt with "Biggest Loser" logo

- District Award Certificates for Highest Percent Loss to each 1st place team within each district, headquarters floor, academy, vice, and animal control.

- $\quad$ District Trophy for the district with most total pounds lost.

- Random Drawing - All teams that completed the program were eligible for a random drawing of one team to win free athletic shoes valued at up to $\$ 100$.

- Grand Prizes - An 8 gigabyte iPod Touch to each team member from top three teams.

The team score was the average percent dropped among all team members. Total value for the awards and prizes was $\$ 6,925$.

\section{Statistical Analysis}

Dropouts were excluded from the data analysis in order to provide a more accurate assessment of the mass lost at each 2 week period. This exclusion potentially affects the ability to accurately assess the overall efficacy of the program. Comparisons between genders and among BMI categories were made relative to the total amount of body mass (weight) lost and amount of change over each 2 weeks of the study. The BMI was calculated $\left(\mathrm{kg} / \mathrm{m}^{2}\right)$ from each participant's initial weigh-in and consisted of the following categories: normal (18.5 to 24.9), overweight (25.0 to 29.9), class I obese (30.0 to 34.9 ), class II obese (35.0 to $39.9)$, and class III obese (>40).

Paired sample T-tests were used to compare starting and final body masses (weights) of all participants, starting and final body masses within each BMI category, and the absolute mass loss within each period. One-way ANOVA tests were used to compare genders in relationship to height, body mass, BMI, and changes that occurred between each successive weigh-in. Also, one-way ANOVA comparisons were used to compare body masses between genders at each weigh-in period. ANOVA with Tukey HSD tests were used to compare body mass changes within BMI categories from start to final weigh-in and the 2-week change at each successive weigh-in. Mean \pm standard deviation (SD) is used to report measures of central tendency and variation. Body weight is reported as body mass in kilograms in the results, tables, and figures to meet the international metric unites standards.

\section{RESULTS}

Out of the 260 police officers that participated in the competition, 225 completed all 7 weigh-ins throughout the 12-week period and were used in this analysis. Approximately $5 \%$ of the officers utilized counseling services for weight loss. There were no

(C) The Internet Journal of Allied Health Sciences and Practice, 2014 
significant differences in age between genders and among BMl categories. The age range was 24 to 55 years with a mean age of $39 \pm 7$ years.

Table 1 is a comparison of police height, body mass, and BMI by gender. As expected, females had significantly $(p \leq 0.001)$ less starting and final body mass. They also had a significantly $(p \leq 0.001)$ lower starting and final BMI. Altogether, the starting body mass was $101.0 \mathrm{~kg}$ and the final body mass was $95.8 \mathrm{~kg}$ with a starting BMl of 32.3 and a final BMl of 30.6 .

Table 1. Comparison of Police Height, Body Mass, and BMI by Gender

\begin{tabular}{|l|l|l|l|l|l|l|l|l|l|l|}
\hline & \multicolumn{3}{|c|}{ Female } & \multicolumn{5}{c|}{ Male } & \multicolumn{3}{c|}{ Total } & \\
\hline & N & Mean & SD & & Mean & SD & N & Mean & SD & p-value \\
\hline Height (cm) & 45 & 166.7 & 7.8 & 179 & 179.2 & 6.9 & 224 & 176.7 & 8.7 & $0.000^{t+t}$ \\
\hline Starting Body Mass (kg) & 46 & 81.9 & 15.8 & 179 & 105.9 & 19.0 & 225 & 101.0 & 20.8 & $0.000^{t t t}$ \\
\hline Final Body Mass (kg) & 46 & 77.6 & 15.0 & 179 & 100.4 & 17.3 & 225 & 95.8 & 19.2 & $0.000^{t+t}$ \\
\hline Starting BMI & 45 & 29.6 & 6.0 & 179 & 32.9 & 5.3 & 224 & 32.3 & 5.6 & $0.000^{t+t}$ \\
\hline Final BMI & 45 & 28.1 & 5.6 & 179 & 31.2 & 4.9 & 224 & 30.6 & 5.2 & $0.000^{t+t}$ \\
\hline
\end{tabular}

ANOVA comparisons between genders: $t+t p \leq 0.001 ; \mathrm{SD}=$ Standard Deviation

Figure 1 portrays the biweekly body mass changes starting with the initial weigh-in. ANOVA comparisons indicated significant body mass loss at each weigh-in period ( $p \leq 0.001)$. There was a decline in the amount of body mass loss starting at the initial weigh-in period with the initial body mass loss being $1.9 \mathrm{~kg}$ and the lowest amount of mass loss being $0.4 \mathrm{~kg}$ at the tenth week weigh-in.

Figure 1. Biweekly Mean Body Mass Change in 12 Week Police Weight Loss Competition

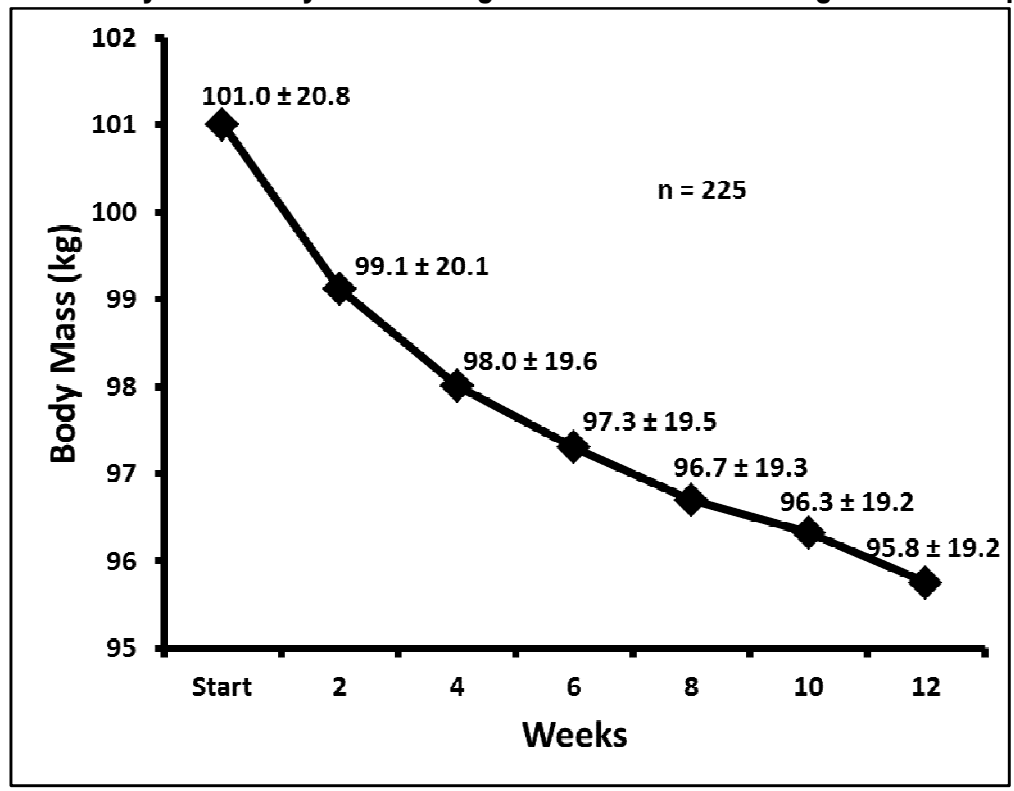

ANOVA comparisons indicate significant body mass loss at each weigh-in period $(p \leq 0.001)$

Mean body mass \pm standard deviation

Figure 2, a gender comparison of body mass loss in the 12-week police weight loss competition, demonstrates no significant differences between genders. There was a declining rate of body mass loss over time with an increase in body mass at the final weigh-in for both genders. Males tended to lose more body mass in the first 5 weigh-in periods, $1.38 \mathrm{~kg}$, and females tended to lose $0.17 \mathrm{~kg}$ more body mass than males at the final weigh-in period. However, the differences were not significant. 
Figure 2. Gender Comparison of Mean Body Mass Loss in 12 Week Police Weight Loss Competition

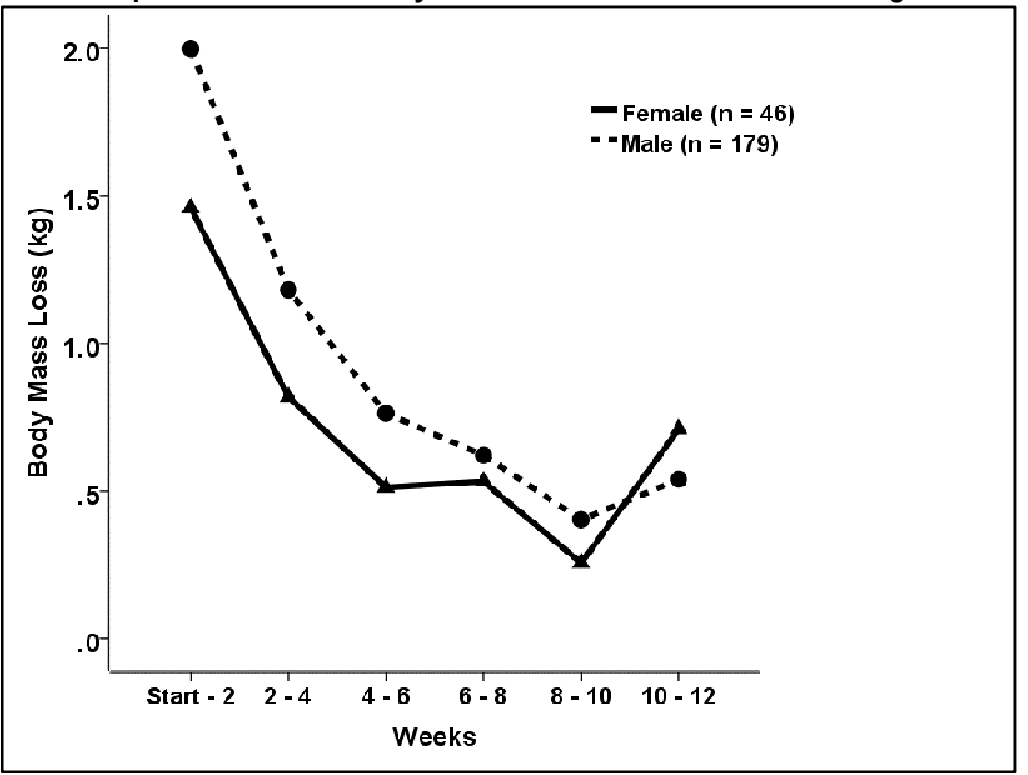

ANOVA comparisons indicated no significant differences between genders

Mean body mass loss \pm standard deviation

Figure 3 illustrates body mass loss from the initial to final weigh-ins among BMl categories over 12 weeks. Those with higher BMls typically lost more body mass. More specifically, the normal and overweight categories lost significantly $(p \leq 0.05)$ less body mass than the 3 obese categories. Furthermore, the extreme obese category lost significantly ( $\leq 0.05)$ more body mass than the 3 lowest BMI categories.

Figure 3. Mean Body Mass Loss from Initial to Final Weigh-In Among BMI Categories Over 12 Weeks

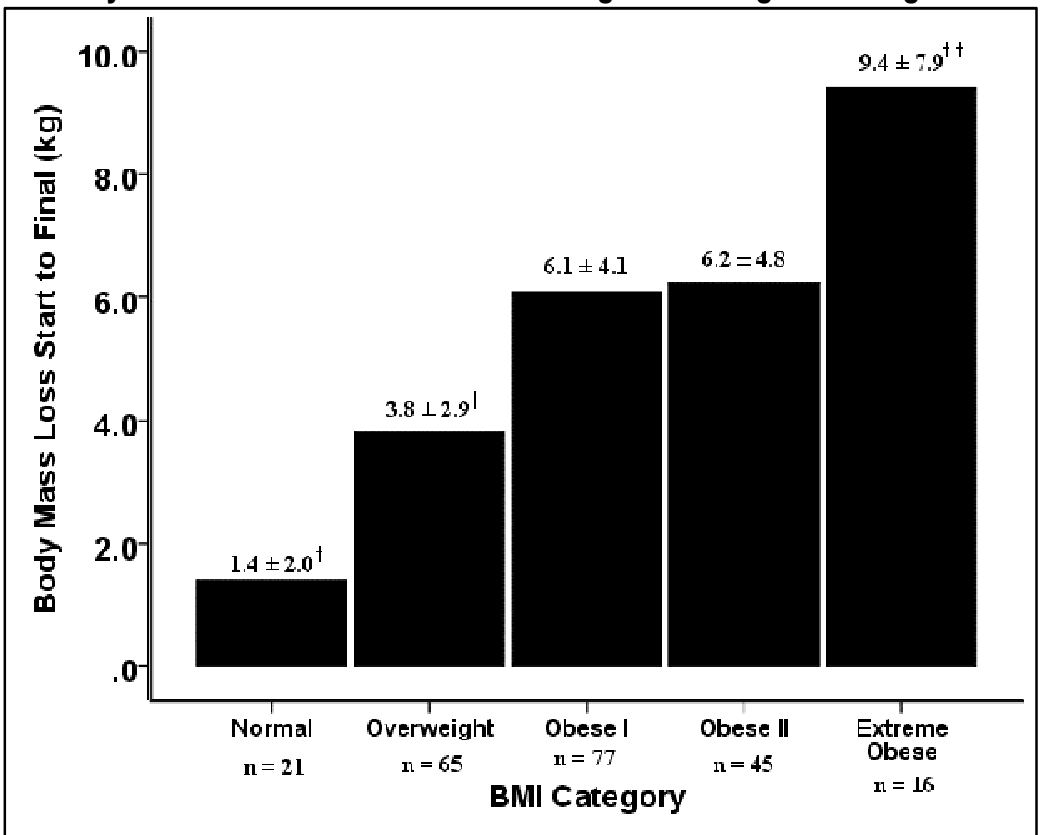

$\dagger$ Normal and overweight are significantly lower than the obese categories $(p \leq 0.05)$

tt Extreme obese is significantly higher than the lowest 3 BMI categories $(p \leq 0.05)$

ANOVA used to compare differences in body mass loss among BMl categories

BMI calculated from initial weigh-in

All BMI categories had a significant $(p<0.01)$ decrease from starting to final weigh-ins

Mean body mass loss \pm standard deviation 
Figure 4 graphically portrays the biweekly mean body mass loss among BMI categories in the 12-week police weight loss competition. The extreme obese category lost more body mass than the other categories in the first 6 weeks. However, this pattern declined from weeks 6 to 12 with no differences found in the amount lost among the groups.

Figure 4. Biweekly Mean Body Mass Loss among BMI Categories in 12 Week Police Weight Loss Competition

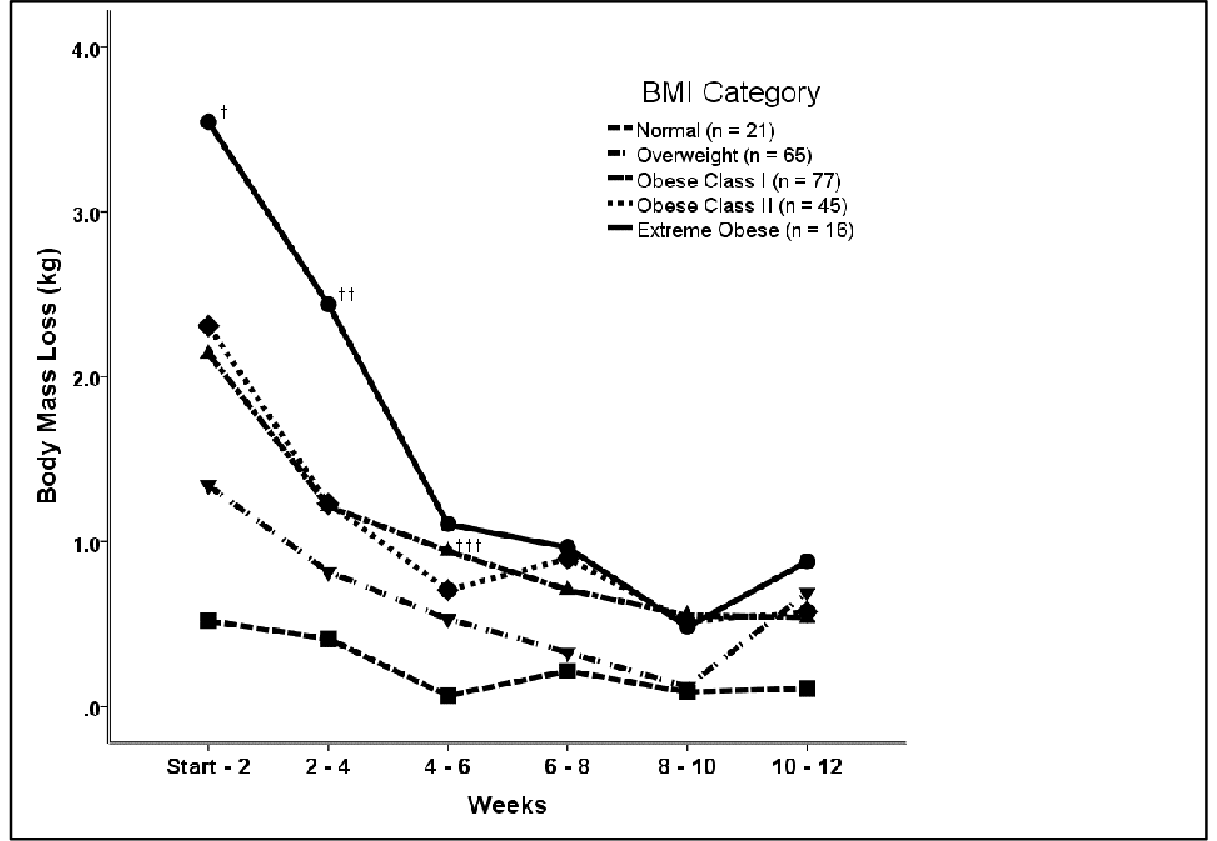

+ Significantly $(p \leq 0.05)$ greater from lowest 3 BMI categories

tt Significantly $(p \leq 0.05)$ greater than all other categories

ttt Significantly $(p \leq 0.05)$ greater than normal category

ANOVA comparisons indicate significant mass loss among noted categories at 2 week intervals

\section{DISCUSSION}

This study was unique from other studies, as it focused solely on police officers participating in a weight loss competition. There were striking similarities in weight loss between genders. An analysis of BMI categories revealed greater mass (weight) loss among those with a higher BMI. In weeks 2 to 4 , the extreme obese lost more body mass than the other BMI categories. However, this relationship diminished from weeks 4 to 12 with little differences among BMI categories from weeks 6 to 12 .

The mean body mass loss was $5.26 \mathrm{~kg}$, which is comparable to other weight loss competition studies. One 12-week competition, among three banks and two industries, $n=277$, yielded an average loss of $5.5 \mathrm{~kg} .{ }^{23}$ Another study involving ten competitions in fifteen worksites, $n=1177$, was also as effective. ${ }^{15}$ In a state-wide internet-based program, Shape Up Rhode Island 2007 (SURI), less weight was lost, $3.2 \mathrm{~kg}$, from individuals completing at least 12 weeks of the 16-week program, $n=6795 .{ }^{24}$

It is interesting to note that even though females had a lower BMI and significantly lower body mass at the start of the competition, the mass loss patterns were similar between genders at each weigh-in period. This is supported by others. ${ }^{15,25}$ The similarity in weight loss between genders in this study may be attributed to similar physical demands within the police occupation. This supports the assumption that differences in worksite physical activities between genders account for different weight loss patterns. ${ }^{26}$

Studies indicate obese people tend to lose weight more rapidly than non-obese or lean people in a small period of time. ${ }^{27,28}$ This is supported further by the positive correlation of initial BMI with weight loss. ${ }^{29}$ While those with the highest BMI lost the most weight during the initial 4 weeks, this trend did not continue. In fact, the rapid losses of the obese categories abated to a rate similar to the lower BMI categories, which exhibited a steadier weight loss pattern throughout the 12-week period. No literature was found concerning law enforcement weight loss initiatives that supported or refuted these findings.

There are numerous strengths to this study. First, there is very little literature that speaks to weight loss programs in police departments. The fact that there were a relatively large number of participants and the attrition rate was low added to the 
statistical strength of the analysis. Also, it provides an opportunity to observe the gender and BMI weight loss patterns within an actual work setting involving participants with similar training, culture, and job demands, thus reducing the complexity of worksite heterogeneity. ${ }^{14}$ It also demonstrated a type of program that can be implemented at low costs and in multiple settings, which is a primary need of police organizations with participants in multiple locations. ${ }^{12}$

The competition team design was effective in this metropolitan police department. This program's success may be a result of the team-oriented police culture as well as the officer's naturally competitive tendencies. Typically, police organizations are predominately male, and it has been reported that male participation increases in weight loss competitions versus individual or cooperative weight loss interventions. ${ }^{15}$ This program and others showed that weight loss differences between genders were minimized making this program especially useful in police organizations, and possibly other related organizations, that need to address obesity issues of both males and females. ${ }^{15}$ Others noted this design, which with easy access to health and fitness professionals, promotes individual and group success. ${ }^{12}$ The exercise physiologist overseeing this weight loss competition indicated that actual use of the fitness professionals was limited to the planning, promoting, and administering of this program, and participants generally did not seek individualized fitness and nutrition prescriptions. It may be that participants in the competition did not take advantage of these services because these resources are routinely available to the police officers during their initial recruit training and regular physical fitness testing.

A primary weakness of this case study was it lacked components of an experimental design such as randomized trials and control groups. Furthermore, there is scrutiny as to the applicability of BMI as an acceptable measure of overweight and obesity classes. ${ }^{30}$ It isn't known if BMl changes were due to regression of the mean or not. Also, there was insufficient information regarding percent body fat, ethnicity, and changes in behavior. Even though the nature of applying this type of intervention requires multiple data collectors at diverse sites, this can increase instrumental and inter-rater variability. There was no postprogram evaluation to determine the participants' perspectives as to the program's effectiveness. Emergency calls and shift changes made it difficult to schedule weigh-ins at the same time of day, which could increase data collection inconsistency.

While numerous weight loss studies exist for other occupations, there is a lack of research regarding law enforcement employees. ${ }^{9}$ Furthermore, efficacy and effectiveness of police weight loss programs should be addressed through experimental design. A unified police initiative to track weight loss longitudinally would enhance understanding and provide a platform for randomized, controlled trials among and within departments. This involves systematic analyses with standard variables such as gender, physical fitness levels, exercise habits, diet, dropout rates, and more definitive measures of body composition. More research is also needed regarding other health measures such as cardiovascular and diabetic risk factors within police settings which could be provided, in part, by personnel medical records. ${ }^{9}$ Reports using standardized questionnaires would be beneficial in providing feedback to ensure systematic improvements such as identifying effective incentives and rewards, improving weight loss outcomes, attendance, motivation, and satisfaction. Finally, it is essential to review and analyze the cost benefits and effectiveness in improving department productivity, morale, and physical fitness.

\section{Suggestions and Recommendations for Applying Findings}

Several recommendations can be made to health education specialists and program coordinators to help achieve optimal outcomes from weight loss programs. These are suggested based partly on the study outcomes and partly our practical observations while conducting the program:

1) The competition format appeared to be highly effective in obtaining and maintaining high levels of participation in appropriate groups.

2) Health education specialists are advised to be prepared with the knowledge of how individual BMI may affect expected weight loss. Those with higher BMI can expect to have the greatest initial weight loss with subsequent losses more similar to other BMI classes in the later part of the competition.

3) The normal BMI participants, according to this data, will probably have a more consistent weight loss throughout the program. Be prepared to provide additional motivation if they get discouraged while observing the more dramatic initial losses of the higher BMI teammates.

4) Be aware that these weight loss patterns are expected in both genders, even if the BMI of the females is generally lower.

5) At the 4 to 6 week mark, adjust the program to provide support when the expected rate of loss typically slows. This is especially important for participants in the obese categories with an emphasis on the extreme obese category.

a. Some possible 4 to 6 week interventions would be:

i. Initiate a "fresh start" program at week 4 to keep everyone motivated, renew commitment, and help them overcome the late program reduction in weight loss.

ii. Provide new exercise routines at the 4 to 6 week mark. Include a greater variety of exercises/activities.

(c) The Internet Journal of Allied Health Sciences and Practice, 2014 
iii. Encourage recommitment to the dietary programming, revisit the diet, and make appropriate individual suggestions.

iv. Inspire renewed commitment with new prizes or bonuses at this phase.

v. Encourage/teach team-building activities to support peer-to-peer coaching to maintain morale and focus on both team and individual goals. This is with the understanding that the regulation of body weight involves complex physiological systems resulting in changes in appetite and metabolic efficiency.

\section{CONCLUSION}

This weight loss competition was a pioneering intervention for police and revealed outcomes and strategies for future police weight management. The findings can be summarized into three major outcomes. First, this case study demonstrated that a competitive, multiple-site, large scale and low cost intervention can be successful for police departments, with the officers themselves assisting in the implementation. Secondly, both males and females were successful, which is crucial in police settings where there has been a history of gender legal issues stemming from hiring and employment practices that adversely impact females. This study suggests a weight loss competition format can equitably address both males and females. Thirdly, although there was a relatively smooth pattern of mass (weight) loss overall, there were striking differences seen in mass (weight) loss behavior across the twelve weeks when observing the different BMI categories. It is interesting to note that after the first half of the program the higher BMI groups did not lose significantly more body mass than the lower BMI groups. This suggests a need for strategies to address these differences mid-way through the intervention. These three outcomes can be translated into practical applications for future health promotion strategies in regards to police departments.

\section{REFERENCES}

1. Flegal KM, Carroll MD, Kit BK, Ogden CL. Prevalence of obesity and trends in the distribution of body mass index among US adults, 1999-2010. JAMA. Feb 1 2012;307(5):491-7. [PMID 22253363]

2. Droyvold WB, Nilsen TI, Kruger O, et al. Change in height, weight and body mass index: Longitudinal data from the HUNT Study in Norway. Int J Obes (Lond). Jun 2006;30(6):935-9. [PMID 16418765]

3. Clinical Guidelines on the Identification. Evaluation, and Treatment of Overweight and Obesity in Adults--The Evidence Report. National Institutes of Health. Obes Res. Sep 1998;6 Suppl 2:51S-209S. [PMID 9813653]

4. Popkin BM, Kim S, Rusev ER, Du S, Zizza C. Measuring the full economic costs of diet, physical activity and obesity-related chronic diseases. Obes Rev. Aug 2006;7(3):271-93. [PMID 16866975]

5. Schulte PA, Wagner GR, Ostry A, et al. Work, obesity, and occupational safety and health. Am J Public Health. Mar 2007;97(3):428-36. [PMID 17267711]

6. Demling RH, DeSanti L. Effect of a Hypocaloric Diet, Increased Protein Intake and Resistance Training on Lean Mass Gains and Fat Mass Loss in Overweight Police Officers. Annals of Nutrition \& Metabolism. 2000;44(1):21-9. [PMID 11333750]

7. Boyce RW, Boone EL, Cioci BW, Lee AH. Physical activity, weight gain and occupational health among call centre employees. Occupational Medicine-Oxford. Jun 2008;58(4):238-44. [PMID WOS:000256283400005]

8. Duncan JTS, Brenner RN, Kravitz M. Police stress: a selected bibliography. Washington, D.C.: U.S. Dept. of Justice, Law Enforcement Assistance Administration, National Institute of Law Enforcement and Criminal Justice: for sale by the Supt. of Docs., U.S. Govt. Print. Off.;1979.

9. Zimmerman FH. Cardiovascular disease and risk factors in law enforcement personnel: a comprehensive review. Cardiol Rev. Jul 2012;20(4):159-66. [PMID 22314143]

10. Baughman P, Fekedulegn D, Andrew ME, et al. Central Adiposity and Subclinical Cardiovascular Disease in Police Officers. ISRN Obesity. 2013;2013:895687. [PMID 24555157]

11. Goetzel RZ, Ozminkowski RJ. What's holding you back: why should (or shouldn't) employers invest in health promotion programs for their workers? N C Med J. Nov-Dec 2006;67(6):428-30. [PMID 17393704]

12. Hennrikus DJ, Jeffery RW. Worksite intervention for weight control: a review of the literature. Am J Health Promot. Jul-Aug 1996;10(6):471-98. [PMID 10163312]

13. Anderson LM, Quinn TA, Glanz K, et al. The effectiveness of worksite nutrition and physical activity interventions for controlling employee overweight and obesity: a systematic review. Am J Prev Med. Oct 2009;37(4):340-57. [PMID 19765507]

14. Benedict MA, Arterburn D. Worksite-based weight loss programs: a systematic review of recent literature. Am J Health Promot. Jul-Aug 2008;22(6):408-16. [PMID 18677881]

15. Stunkard AJ, Cohen RY, Felix MR. Weight loss competitions at the worksite: how they work and how well. Prev Med. Jul 1989;18(4):460-74. [PMID 2798370]

16. Ostbye T, Dement JM, Krause KM. Obesity and workers' compensation: results from the Duke Health and Safety Surveillance System. Arch Intern Med. Apr 23 2007;167(8):766-73. [PMID 17452538] 
17. Kelly KL. Evaluation of a Group Nutrition Education Approach To Effective Weight Loss and Control. Am J Public Health. 1979;69(8):813-4. [PMID 453416]

18. Williamson DF, Serdula MK, Anda RF, Levy A, Byers T. Weight loss attempts in adults: goals, duration, and rate of weight loss. Am J Public Health. Sep 1992;82(9):1251-7. [PMID 1503167]

19. Gorin AA, Marinilli Pinto A, Tate DF, Raynor HA, Fava JL, Wing RR. Failure to meet weight loss expectations does not impact maintenance in successful weight losers. Obesity(Silver Spring). Dec. 2007;15(12):3086-90. [PMID 18198318]

20. Hersey J, Williams-Piehota P, Sparling PB, et al. Promising practices in promotion of healthy weight at small and mediumsized US worksites. Prev Chronic Dis. Oct 2008;5(4):A122. [PMID 18793510]

21. Briley ME. Effects of Nutritional Program on Weight Loss and Lipid Profile of Police Department Employees Phase I. Federation Proceedings. 1987;46(4).

22. U.S. Department of Health and Human Services. Healthy People 2020. http://www.healthypeople.gov/2020/topicsobjectives2020/pdfs/HP2020objectives.pdf. Accessed July 24, 2012.

23. Brownell KD, Cohen RY, Stunkard AJ, Felix MR, Cooley NB. Weight loss competitions at the work site: impact on weight, morale and cost-effectiveness. Am J Public Health. Nov 1984;74(11):1283-5. [PMID 6437259]

24. Wing RR, Pinto AM, Crane MM, Kumar R, Weinberg BM, Gorin AA. A statewide intervention reduces BMI in adults: Shape Up Rhode Island results. Obesity (Silver Spring). May 2009;17(5):991-5. [PMID 19180068]

25. Linde JA, Jeffery RW, Levy RL, Pronk NP, Boyle RG. Weight loss goals and treatment outcomes among overweight men and women enrolled in a weight loss trial. Int J Obes (Lond). Aug 2005;29(8):1002-5. [PMID 15917847]

26. Droyvold WB, Holmen J, Midthjell K, Lydersen S. BMI change and leisure time physical activity (LTPA): an 11-y follow-up study in apparently healthy men aged 20-69 y with normal weight at baseline. Int J Obes Relat Metab Disord. Mar 2004:28(3):410-7. [PMID 14724661]

27. Lejeune MP, Van Aggel-Leijssen DP, Van Baak MA, Westerterp-Plantenga MS. Effects of dietary restraint vs exercise during weight maintenance in obese men. Eur J Clin Nutr. Oct 2003;57(10):1338-44. [PMID 14506498]

28. Stein PM, Hassanein RS, Lukert BP. Predicting weight loss success among obese clients in a hospital nutrition clinic. Am J Clin Nutr. Oct 1981;34(10):2039-44. [PMID 6270997]

29. Handjieva-Darlenska T, Handjiev S, Larsen TM, et al. Initial weight loss on an $800-k c a l$ diet as a predictor of weight loss success after 8 weeks: the Diogenes study. Eur J Clin Nutr. Sep 2010;64(9):994-9. [PMID 20588292]

30. Mills TC, Gallagher D, Wang J, Heshka S. Modelling the relationship between body fat and the BMI. Int J Body Compos Res. 2007;5(2):73-9. [PMID 22049264]

\section{KEY TERMS}

Body Weight, Law Enforcement, Quetelet Index, BMI, Adult, Intervention, Health Promotion 